\title{
Quelques exemples d'avant-projets d'accumulation pneumatique
}

\author{
par 6. Rigollot \\ Ingénieur-Conseil, \\ Société Générale d'Etude et d'Equipement, Paris
}

$$
-\mathrm{I}-
$$

On sait - et les orateurs qui nous ont précédé viennent de le rappeler - que l'accumulation pneumatique pourra utiliser différents types de réservoirs:

- Cavernes creusées dans des gisements de sel;

- Anticlynaux poreux ou fissurés, sous couverture étanche et aquifère actif;

- Cavernes minées, créées dans du rocher (granit et par apparentement calcaire) ou dans des terrains sédimentaires consolidés (craie);

- On pourrait même, pour certaines applications particulières, faire appel à des réservoirs métalliques de surface.

On sait aussi que l'accumulation de l'air comprimé de même que l'opération inverse de prélèvement peuvent être réalisées :

- Soit à pression constante, grâce à la compensation hydraulique;

- Soit à pression variable, par utilisation d'un volume d'air coussin;

- Soit même - pourquoi pas - par superposition des deux modes de fonctionnement.

Dans tous les cas, la pression à la turbine est maintenue pratiquement constante, au besoin par laminage, sans que ce dernier, même important, ne provoque de pertes inacceptables de rendement. On pourrait, d'ailleurs, si nécessaire, moduler la pression d'admission pour permettre un ajustement modéré de la puissance émise sur le réseau.

Les usines de production d'énergie avec accumulation pneumatique peuvent, en outre, être conçues de plusieurs manières :

- Suivant un schéma simple (fig. 1) avec détente de l'air comprimé après chauffage;

- En profitant de l'amélioration déjà escomptée pour les turbines à gaz classiques, par accroissement de l'énergie produite, à quantité d'air utilisé égale, grâce à des injec- tions d'eau ou de vapeur d'eau, dans la chambre de combustion de la turbine;

- En association avec des cycles vapeur traditionnels ou de type nouveau pour constituer des ensembles de très grande puissance à consommation spécifique globale et coûts d'équipements et d'exploitation avantageux.

De plus, et à condition de suivre une évolution technologique méthodique et raisonnable, on pourra :

- Choisir avec une certaine liberté les températures et pres. sions d'admission et les débits massiques respectifs du compresseur et de la turbine;

- Utiliser les ressources de la rechauffe et de la récupération partielle de la chaleur des gaz d'échappement.

Il s'ensuit que l'on disposera d'une grande variété d'installations:

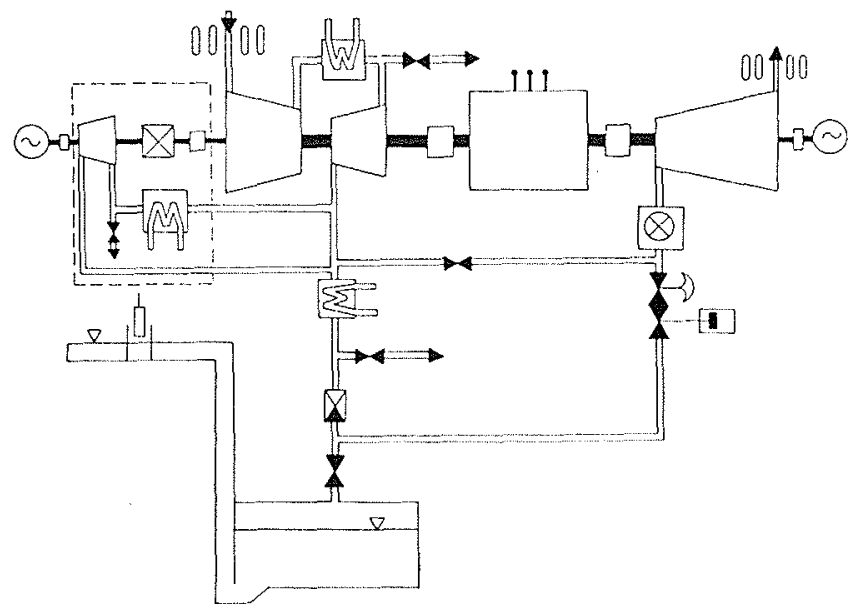

1/ 


\section{G. RIGOLLOT}

- Qui auront entre elles un certain nombre de points communs essentiels :

- puissance unitaire élevée (supérieure à $200 \mathrm{MW}$ );

- durée de production journalière programmée;

- grande facilité de démarrage et d'arrêt;

- bonne adaptation à la production d'énergie de complément, semi-base et pointe);

- Mais qui différeront profondément entre elles sur d'autres points :

- performances techniques et consommation de combustible; ou d'énergie de compression;

- durée d'utilisation journalière;

— coûts d'équipement;

- localisation et possibilités d'implantation.

\section{- II -}

Au moment donc d'examiner quelques aspects de la conception d'avant-projets d'installation de production d'énergie à accumulation pneumatique, il a paru utile, pour mieux illustrer cet exposé, de définir une méthode simple de comparaison permettant de mieux juger de l'intérêt de chacun d'eux.

Si l'on admet que l'énergie de complément n'est pas forcément une énergie chère et que la production avec accumulation - en particulier pneumatique - a justement pour but d'en ramener le coût à des valeurs aussi proches que possible de celui de l'énergie de base, il est dès lors naturel de prendre ce dernier comme terme de comparaison.

- On se placera dans l'optique sinon peut-être de la prochaine décennie, du moins de la suivante pour laquelle l'énergie de base susceptible d'être transférée sera d'origine nucléaire;

- Mais on laissera, toutefois, de côté deux postes de dépenses sur lesquels le technicien a d'ailleurs moins d'influence : - les charges d'exploitation qui comportent une part fiscale non négligeable et qui dépendent, en outre, des structures internes des sociétés d'électricité;

- les charges de transport d'énergie, fonctions, elles, de circonstances locales propres à chaque région ou pays;

- On ne chiffrera pas non plus l'avantage réel offert par l'accumulation pneumatique et résultant de sa souplesse de démarrage et d'arrêt, ni celui particulier à certains schémas de mise à disposition instantanée d'une notable puissance de secours, ni enfin de celui résultant de la possibilité de faire fonctionner, si besoin est, l'installation thermique en turbine à gaz traditionnelle, en dehors des périodes de mise en stock et de prélèvement.

- Et on prendra seulement en considération les dispositions du tableau 2 soit:

- les dépenses de capital;

- les dépenses d'énergie calorifique du fuel ajouté et électrique de compression pour l'accumulation de l'air;

- les dépenses afférentes au stock stratégique de sécurité du fuel.

En ce qui concerne ce clernier point, les événements mondiaux qui viennent de se dérouler ont, en effet, conduit les pays ne possédant pas de réserves de combustible fossile sur leur sol propre, à s'orienter vers un recours préférentiel sinon exclusif à l'énergie nucléaire, restant toutefois tributaires du pétrole pour la production d'énergie de complément, eu égard à des considérations économiques et plus encore pour bénéficier d'une bonne souplesse d'exploitation. Mais la sécurité de l'irrigation d'un pays en énergie électrique nécessite, alors, la construction de stocks stratégiques de couverture, affectés en quelque sorte à chaque usine thermique et dont le coût total - contenant et contenu doit intervenir dans le calcul comparatif du prix de revient de l'énergie.

Le tableau 1 rappelle les éléments et hypothèses qui ont été pris en compte ici, mais qui restent évidemment soumis au réexamen et à l'appréciation des différents spécialistes intéressés.

A noter que dans l'évaluation du coût de l'énergie produite par l'accumulation pneumatique, il a été supposé que :

- L'énergie nocturne emmagasinée est entièrement d'origine nucléaire au prix de $1 \mathrm{c} / \mathrm{kWh}$;

- Le prix de l'énergie calorifique alimentant les chaudières à vapeur des cycles mixtes et provenant de fuel traditionnel est de $0,9 \mathrm{c} /$ thermie;

- L'énergie calorifique du fuel ajouté dans la turbine à gaz coûte, en raison de la qualité particulière de ce dernier, $15 \%$ de plus que la précédente.

$$
-\mathrm{III}-
$$

Le premier des avant-projets présentés aujourd'hui, a trait à l'utilisation d'anticlynaux poreux sous aquifère actif.

C'est d'ailleurs ce type de réservoir qui avait été pris en considération lors des premières études entreprises dès 1951 par l'Electricité de France sur l'accumulation pneumatique et que nous évoquions l'autre jour avec M. Réménieras.

En 1966, sous l'impulsion de MM. Banal, Robin et de Villeneuve, une étude méthodique fut entreprise en choisissant comme exemple concret la structure assez bien connue du pays de Bray. Celle-ci se situe à une profondeur moyenne de $250 \mathrm{~m}$ et comporte une couche en sable de glos d'une hauteur variant de 10 à $50 \mathrm{~m}$. La perméabilité en est assez réduite, environ 0,3 darcy, mais cette modeste valeur ne constitue pas le défaut de cette formation: l'influence défavorable n'en est sensible qu'aux abords immédiats des puits et l'on pourrait envisager de la pallier par divers moyens d'amélioration locale. C'est la présence de deux failles sur le flanc nord-est de l'anticlynal qui fait peser un doute sur l'utilisation de cette structure, l'étanchéité satisfaisante du réservoir ne pouvant être vérifiée avant mise en air complète et, pratiquement, achèvement de tous les travaux.

Il existe certainement en France des structures plus profondes, de meilleure perméabilité et présentant une bonne continuité de la couverture d'étanchéité.

Mais le mérite de cette étude prenant en compte des hypothèses relativement peu favorables fut de mettre clairement en évidence, l'existence des divers facteurs intervenant dans le dimensionnement des installations de ce type et le choix des caractéristiques et d'en mesurer l'influence.

On résumera sommairement les enseignements tirés de cette étude par les commentaires suivants :

- Constitué par les espaces interstitiels entre grains de sable, le réservoir proprement dit ne coûte rien: il est dès lors possible de prévoir de longues utilisations journalières en pratiquant la compensation dite hebdomadaire par accumulation d'énergie durant les heures de faible charge électrique de fin de semaine;

- Ce sont, au contraire, les organes d'injection et de reprise d'air comprimé - en l'occurence les puits - en nombre 


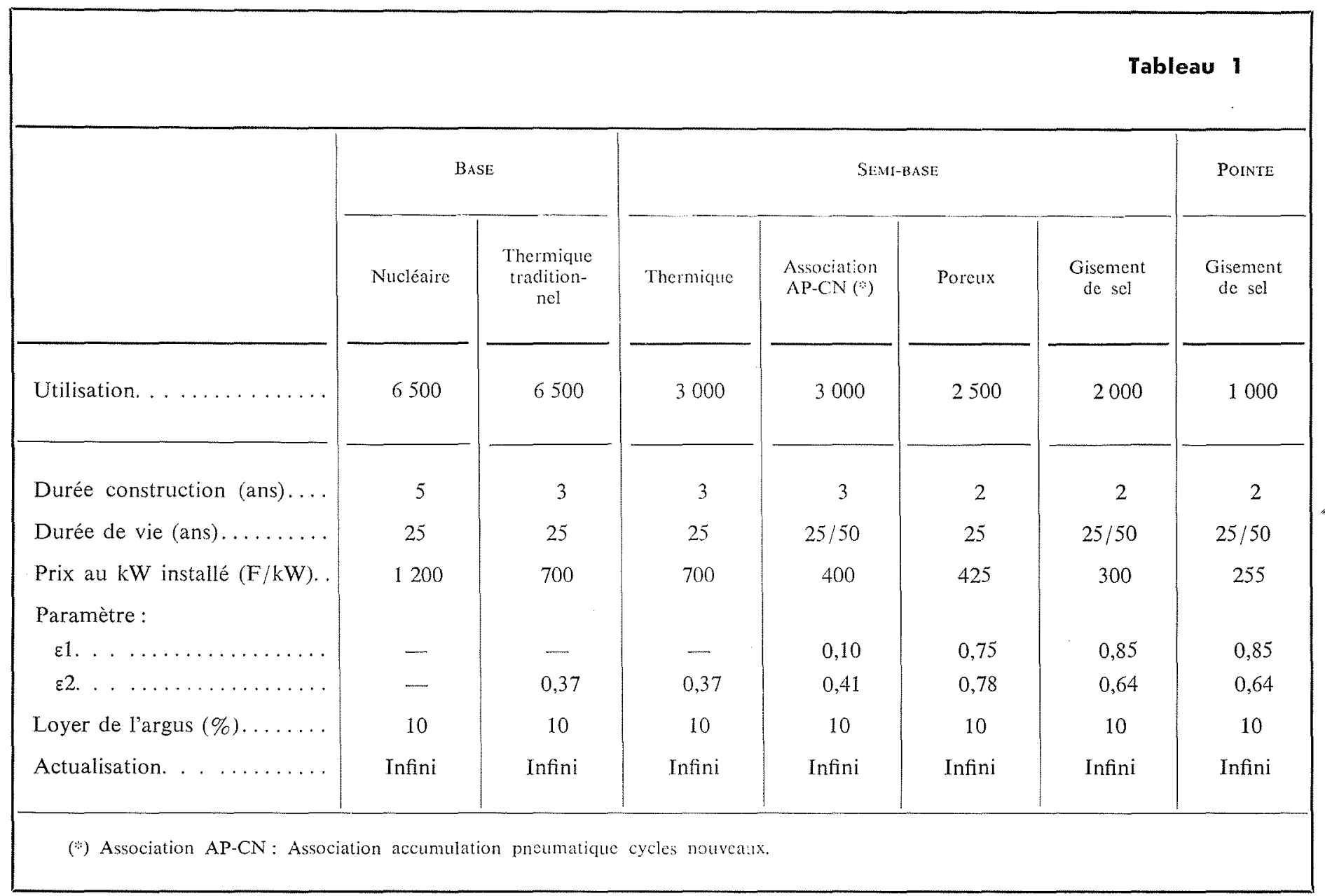

\begin{tabular}{|c|c|c|c|c|c|c|c|}
\hline & & & & & & \multicolumn{2}{|c|}{ Tableau 2} \\
\hline & \multicolumn{2}{|c|}{ BASE } & \multicolumn{4}{|c|}{ SEMI-BASE } & \multirow{2}{*}{$\begin{array}{c}\text { PoINTE } \\
\begin{array}{c}\text { Gisement } \\
\text { de sel }\end{array}\end{array}$} \\
\hline & Nucléaire & $\begin{array}{c}\text { Thermique } \\
\text { tradition- } \\
\text { nel }\end{array}$ & Thermique & $\begin{array}{l}\text { Association } \\
\text { AP-CN }(*)\end{array}$ & Poreux & $\begin{array}{l}\text { Gisement } \\
\text { de sel }\end{array}$ & \\
\hline Utilisation. . . & 6500 & 6500 & 3000 & 3000 & 2500 & 2000 & 1000 \\
\hline \multirow{4}{*}{$\begin{array}{l}\text { Capital. . . . . . . . . . . . . . . . } \\
\text { Combustible. . . } \\
\text { Stockage stratégique...... }\end{array}$} & 2,49 & 1,35 & 2,92 & 1,65 & 2,13 & 1,77 & 2,79 \\
\hline & 1,00 & 1,98 & 1,98 & 2,04 & 1,89 & 2,23 & 2,23 \\
\hline & - & 0,78 & 0,78 & 0,67 & 0,19 & 0,23 & 0,23 \\
\hline & 3,49 & 4,11 & 5,68 & 4,36 & 4,21 & 4,23 & 5,25 \\
\hline
\end{tabular}

(s) Association AP-CN : Association accumulation pncumatique cycles nouveaux. 


\section{G. RIGOLLOT}

proportionnel à la puissance des unités desservies - qui déterminent le montant de l'investissement extérieur au bloc usine. On sera donc conduit à pratiquer des débits unitaires très élevés, en s'approchant au plus près des valeurs limites de vitesse imposées par l'entraînement de particules, l'érosion dans les tubes de remontée, la stabilité des crépines et des parties adjacentes de la formation. Cependant, en dépit d'une utilisation intensive des puits, leur nombre est élevé (environ un puits pour deux mégawatts);

- Compte tenu des interférences entre puits, on est conduit à répartir ceux-ci en groupes de quatre, branchés sur une même ramification du réseau de collecte, ces groupes formant par ailleurs un quadrillage régulier couvrant toute la partie utilisée de la structure;

- Si, en surface, on se trouve en région habitée ou en zone d'exploitation rurale, un problème d'environnement se pose qui s'ajoute à celui de l'approvisionnement en combustible ou en eau de réfrigération, la structure pouvant se trouver relativement éloignée des axes de communication ferroviaire ou des points possibles de prise d'eal d'appoint.

- Ces sujétions ne seront en général tolérées que par des usines de grande puissance unitaire (par exemple deux groupes de $250 \mathrm{MW}$ ) et tirant le meilleur parti énergétique possible de l'air stocké, c'est-à-dire utilisant simultanément

- des pressions et températures élevées à l'admission de la turbine;

- la double chauffe;

- la récupération de chaleur sur les gaz d'échappement;

- la superposition des deux processus mentionnés cidessus, à compensation hydraulique partielle et à coussin pneumatique tampon, en faisant fonctionner la bulle d'air à volume et pression variables — si l'on peut toutefois appeler bulle le volume limité par l'interface eau-air, à faciès tourmenté témoin de l'hétérogénéité du banc et des variations locales de perméabilité;

- L'injection d'eau dans la chambre de combustion, dont il n'a pas été tenu compte dans les calculs, apporterait une amélioration très sensible à l'économie du projet

Quoi qu'il en soit, dans le cas du pays de Bray, l'usine à deux groupes de $250 \mathrm{MW}$, à utilisation journalière de 12 à $14 \mathrm{~h} / \mathrm{j}$ et à consommation spécifique caractérisée par:

$$
\begin{aligned}
& \varepsilon 1=0,75 \\
& \varepsilon 2=0,78
\end{aligned}
$$

coûterait sur les bases économiques actuelles $425 \mathrm{~F} / \mathrm{kW}$ installé aux bornes de sortie des transformateurs, avec des services généraux d'usine allégés mais suffisants pour l'administration et la maintenance.

A ces performances et caractéristiques correspondent un coût de l'énergie produite de $4,21 \mathrm{c} / \mathrm{kWh}$ dont la décomposition poste par poste figure dans la cinquième colonne du tableau 2 (utilisation $2500 \mathrm{~h} / \mathrm{an}$ ).

De conception et d'essence différentes, se présente le second des avant-projets examinés au cours de cet exposé. Il s'agit de l'accumulation pneumatique dans des cavités créées dans un gisement de sel.
C'est le type le meilleur marché de réservoir et celui qui présente le minimum d'aléas; à condition que la structure salifère ait été convenablement reconnue, l'étanchéité et la stabilité des cavernes sont pratiquement assurées. Les forages d'injection et de prélèvement d'air qui ont déjà servi préalablement à l'exécution, par voie hydraulique, des volumes souterrains sont ensuite équipés pour assurer les échanges d'air entre la caverne et la surface.

C'est précisément l'évacuation des saumures provenant de la dissolution du sel qui constitue le problème majeur. Leur volume est sensiblement décuple de celui des cavités créées et leur évacuation peut s'effectuer :

- dans une rivière ou un fleuve à condition que celui-ci ait un débit d'étiage suffisant; mais l'autorisation de rejet sera en général difficile à obtenir;

- à la mer ou dans des étangs reliés à la mer;

- dans les couches profondes des terrains si la géologie locale est favorable;

- à travers une industrie chimique de transformation suivant circonstances particulières.

Il faut noter toutefois que :

- L'accumulation pneumatique en gisement de sel pourra avantageusement se trouver associée à la création des stockages d'hydrocarbures stratégiques nationaux, où elle bénéficiera alors de conditions de réalisation privilégiées;

- Même s'il n'en est pas ainsi, la modeste valeur des volumes à créer $\left(300000 \mathrm{~m}^{3}\right.$ environ par unité de $200 \mathrm{MW}$ pour utilisation journalière de $8 \mathrm{~h} / \mathrm{j}$ ) autorise des vitesses de création et corrélativement des débits d'évacuation des saumures faibles ce qui facilitera grandement la solution de ce problème.

La production d'énergie avec accumulation pneumatique dans des cavités creusées dans des gisements de sel présente les particularités suivantes:

- Elle s'effectuera suivant le processus à pression variable, sur volume d'air coussin; les diamètres usuels de forage ne permettent pas aux débits pratiqués le transfert simultané de l'air et de la saumure de compensation.

- Le coût peu élevé de création du volume permet d'accepter un certain nombre de sujétions:

- écart raisonnable de fluctuations de pression pour faciliter la marche en régime variable du compresseur;

- variations de la température moyenne dans la cavité à la mise en stock et au prélèvement;

tout en s'accommodant de turbines à moyenne pression ( 25 bars environ), sans rechauffe, à température modérée d'admission $\left(800^{\circ} \mathrm{C}\right)$, ce qui dispense corrélativement de procéder à une récupération systématique de chaleur sur les gaz d'échappement, la température naturelle d'émission de ceux-ci $\left(270^{\circ} \mathrm{C}\right)$ étant déjà suffisamment basse.

Cette formule d'accumulation conduit donc à des installations simples, à rendement honorable:

$$
\begin{aligned}
& \varepsilon 1=0,85 \\
& \varepsilon 2=0,64
\end{aligned}
$$

et à un prix au $\mathrm{kW}$ installé très intéressant.

On peut concevoir deux variantes d'installation à partir des mêmes compresseurs basse et moyenne pression, de débit nominal standard $(350 \mathrm{~kg} / \mathrm{s})$.

- La première produisant $200 \mathrm{MW}$ pendant $8 \mathrm{~h} / \mathrm{j}$ (utilisation annuelle $2000 \mathrm{~h} / \mathrm{an}$ ) d'un prix au $\mathrm{kW}$ installé bornes transformateur de $300 \mathrm{~F} / \mathrm{kW}$. 
- La seconde produisant $400 \mathrm{MW}$ pendant $4 \mathrm{~h} / \mathrm{j}$ (utilisation annuelle $1000 \mathrm{~h} / \mathrm{an})$ d'un prix au $\mathrm{kW}$ installé bornes transformateur de $235 \mathrm{~F} / \mathrm{kW}$.

On peut même en accouplant deux turbines identiques de $200 \mathrm{MW}$ installées à des époques successives passer de l'une à l'autre variante en fonction de l'évolution des besoins de pointe pure; cette option est envisagée très sérieusement dans l'avant-projet scandinave aux études préliminaires desquelles nous sommes actuellement associés.

L'application de ces paramètres énergétiques et économiques au modèle de calcul du tableau 2 montre que l'on peut obtenir des coûts d'énergie de semi-base proches de celui du nucléaire de base et des coûts d'énergie de pointe qui ne sauraient être approchés par aucun des moyens traditionnels.

$$
-\mathrm{V}-
$$

Nous pensons pouvoir affirmer, bien que nous en ayons poussé moins loin l'étude d'avant-projet qu'une installation suivant le même schéma mais utilisant un réservoir creusé dans un bon rocher (granit ou calcaire) à des profondeurs raisonnables, et avec compensation hydraulique, donnerait encore - pour une utilisation de 1000 à $1200 \mathrm{~h} / \mathrm{an}$ - de bons résultats, surtout si la région est déficitaire en énergie aux charges élevées du réseau.

On se trouve donc en présence de deux types d'avantprojets sérieux, pouvant susciter des réalisations rapides, et dont il peut paraître logique de pousser les études exploratoires en parallèle.

$$
-\mathrm{VI}-
$$

Le dernier chapitre, pour terminer, sera consacré à une analyse prévisionnelle de l'accumulation pneumatique dans des cavités creusées en terrains sédimentaires, plus ou moins consolidés.

C'est évidemment la forme d'accumulation la plus intéressante. En effet, dans les trois avant-projets précédents, l'asservissement de l'implantation des unités à la géologie régionale est totale. Or, si l'on veut dans les deux ou trois décennies à venir et avant que d'autres moyens de production d'énergie ne soient découverts et mis en ouvre, profiter pleinement des avantages économiques offerts par l'accumulation pneumatique, il faudrait que la localisation des usines de production puisse être choisie librement, sans référence au relief ou à la géophysique, en fonction seulement des besoins à satisfaire.

Or, dans le monde et en France notamment, les zones fortement industrialisées, grandes consommatrices d'énergie, sont en général implantées, non sur des socles granitiques, mais sur des terrains sédimentaires à caractéristiques mécaniques moins favorables dont devront cependant s'accommoder les réservoirs de stockage d'air des installations de production chargées d'alimenter ces régions en énergie électrique de complément.

Mais c'est la forme d'accumulation la plus difficile à réaliser, bien que les spécialistes des travaux souterrains affirment que la construction de tels réservoirs dans de tels terrains, et sans revêtement continu, soit toujours possible dès lors que la cohésion en profondeur des couches sédimentaires est suffisante.

Le prix au $\mathrm{m}^{3}$ de telles cavités est toutefois élevé, souvent plus du double de celui que l'on rencontrerait dans des formations de bon rocher, et bien qu'aux valeurs de volume nécessaire, l'amortissement du coût très élevé des ouvrages d'accès en profondeur paraisse pouvoir être correctement assuré.

Il convient donc d'utiliser cette forme d'accumulation avec discernement en tenant compte que dans ces zones à forte consommation la forme des courbes de charge journalière laisse souvent apparaître d'importants besoins diurnes (12 à $14 \mathrm{~h} / \mathrm{j}$ ) correspondant à une utilisation annuelle de 3000 à $3500 \mathrm{~h} / \mathrm{an}$.

C'est pour répondre à ces différents impératifs - liberté de localisation - longue durée diurne d'utilisation - création coûteuse de cavernes minées en terrain sédimentaire consolidé - que nous avons poussé l'étude de formes plus compliquées d'accumulation pneumatique offrant une valorisation énergétique aussi élevée que possible du volume de stockage, ce qui, ajouté notamment aux possibilités offertes par :

- La conjugaison des processus de fonctionnement à compensation hydraulique et à pression variable;

- L'utilisation des basses températures de stockage de l'air et l'injection d'eau ou de vapeur d'eau à la chambre de combustion de la turbine, en profitant des perspectives que cette dernière technique ouvre à la turbine à gaz traditionnelle;

- L'adoption de réfrigération accentuée de l'air en cours de compression;

- Le choix de caractéristiques plus élevées de pression et de température d'admission;

- Le recours à la rechauffe en cours de détente;

est réalisé très heureusement par l'association de l'accumulation pneumatique à des cycles mixtes nouveaux à vapeur, avec prélèvement et compression simultanés, schéma dont nous avons, il y a deux ans, jeté les premières bases avec le S.E.P.T.E.N. et en particulier avec MM. Mascarello et Charrier.

$\mathrm{Au}$ stade actuel de cette étude exploratoire, les schémas demeurent relativement compliqués, mais de nombreuses simplifications apparaissent déjà et en faisant un choix judicieux et cohérent parmi les divers facteurs d'amélioration rappelés brièvement ci-avant, et surtout en les utilisant à plusieurs fins, on peut être certain que l'on parviendra à créer des unités à cycle mixte et accumulation pneumatique de 1000 à $1200 \mathrm{MW}$ de puissance continue disponible $12 \mathrm{~h} / \mathrm{j}$ (utilisation annuelle $3000 \mathrm{~h} / \mathrm{an}$ ) capables, en outre, de libérer instantanément une surpuissance de secours de $150 \mathrm{MW}$ environ, susceptible d'être maintenue pendant une demiheure environ.

L'inspection des performances économiques prévisibles dans le modèle de calcul comparatif utilisé pour cet exposé, conduit à un coût d'énergie à moyenne utilisation extrêmement favorable, même sans tenir compte de l'avantage, difficilement chiffrable, mais très important, offert par la possibilité de surcharge mentionnée ci-dessus.

$$
\text { - VII - }
$$

On voit done que l'application de l'accumulation pneumatique à des terrains sédimentaires devrait être prise en considération et cela d'autant plus que l'amélioration des moyens technologiques d'exécution des ouvrages de génie civil est certaine. 


\section{G. RIGOLLOT}

En ce qui concerne précisément la technologie, on indiquera pour terminer ce rapide tour d'horizon, que les divers avant-projets d'unités de production d'énergie avec accumulation pneumatique, dont nous venons de donner succinctement quatre exemples, font tous appel à des éléments connus, mais qui - dans la plupart des cas — n'ont pas été employés simultanément sur la même installation.

C'est pourquoi, si le développement de l'accumulation doit voir le jour, on peut penser qu'il y aura deux générations d'usines et de matériel.

- La première correspondra à l'utilisation de réservoir bon marché (y compris les dispositifs de mise en stock et de prélèvement). Les schémas thermiques seront simples, le matériel de caractéristiques moyennes. Ces installations à rentabilité immédiate permettront de vérifier la mise en harmonie des différentes technologies en présence et de dégager les critères d'exploitation de ces usines d'un type nouveau. Elles conviendront bien à des utilisations inférieures à $2000 \mathrm{~h} / \mathrm{an}$;
- La seconde, qui devra voir le jour quand l'énergie nucléaire aura conquis sa place, permettra en faisant appel à des schémas et à du matériel plus sophistiqués et bien adaptés, de prendre une place appréciable dans les zones d'utilisation $2500-3000 \mathrm{~h} / \mathrm{an}$.

La première catégorie d'installations se rapporte à un proche futur constructif; la seconde à un avenir où le développement des besoins et le manque de souplesse des moyens de production de base rendront indispensables la création spéciale d'usines économiques à moyenne utilisation, destinées à être installées aux points névralgiques des réseaux de distribution à très haute tension.

Nota: Définition :

$\varepsilon 1$ est le rapport de l'énergie électrique de compression et de l'énergie produite.

$\varepsilon 2$ est le rapport de l'énergie produite et de l'énergie calorifique ajoutée.

\section{Abstract \\ Typical examples of pneumatic energy-storage schemes}

I. Owing to the possible use of various kinds of reservoir, operating schemes and types of equipment, compressed-air storage lends itself to use in a great variety of scheme types with widely differing characteristics, costs and siting possibilities.

II. The paper describes a simple economic model by means of which the advantages and fields of application of the several draftschemes presented are assessed. These are capable of providing peak-demand power at a cost only marginally higher than at the normal level.

III. Draft-schemes presented include the following:

(a) The initial studies were based on storage in porous rock.

(b) Cavities created in salt deposits appear, however, to afford the most economical and the best understood solution at the present time. (c) Cavities dynamited out of granite or limestone also appear to ofler a satisfactory solution from the economic standpoint provided rock quality and local grid conditions create no obstacle.

(d) Artificial reservoirs in sedimentary terrain (particularly chalk) will be needed so that air-storage schemes may be sited where they are wanted, i.e. in areas of heavy power consumption. The higher cost of these may be acceptable in the case of a new type of very high power plant supplying a moderate amount of energy. In such plant air storage would be associated with a special type of steam cycle.

1V. The first generation of air-storage schemes will be provided by schemes (b) and (c) above whereas (a) and (d) will provide the second. The second generation will spread over the next two or three decades and will benefit from teething troubles and experience with the earlier, simpler type of plant. 


\section{DISCUSSION DES COMMUNICATIONS}

M. J. RAUD :

PROBIÈMES DE GÉNIE CIVIL CONCERNANT QUELQUES TYPES D'INSTALLATIONS PNEUMATIQUES DE TRANSFERT D'ÉNERGIE

M. Ch. B. Roux :

L'EXPÉRIENCE DU "GAZ DE FRANCE" EN MATIÈRE DE RÉSERVOIRS SOUTERRAINS DE GAZ COMPRIMÉS

\section{G. Rigollot:}

QUELQUES EXEMPLES D’AVANT-PROJETS D'ACCUMULATION PNEUMATIQUE

Au terme de ces trois exposés, M. le Président, après avoir remercié chaleureusement les conférenciers, ouvre la discussion sur l'ensemble; il propose de l'articuler comme suit:

a) Réservoirs; aménagement; exploitation;

b) Cycles thermodynamiques et technologie des turbines à gaz d'aujourd'hui; perspectives d'avenir;

c) Coût et rentabilité des usines d'accumulation par pompage pneumatique.

a) RÉservotrs de STOCKage d'air COMPRimé:

M. le Président pose à $M$. Roux les questions ci-après :

M. Roux nous a dit que Gaz de France avait reconnu de nombreux réservoirs a aménager dans les nappes aquifères ou des couches de sel. Est-ce que, à vue humaine, ces réservoirs aquifères doivent être réservés en tolalité à Gaz de France ou certains d'entre cux peuvent-ils être hitilisés par Electricité de France?

Je ne saurai dire que tous les réservoirs, connus ou à découvrir, doivent être réservés à Gaz de France, répond $M$. Roux, mais il est bon de remarquer que la puissance énergétique déliviéc par un puits d'exploitation d'un stockage en nappe aquifère est, pour le gaz naturel, cinquante fois supérieure à celle qu'on pourrait obtenir avec de l'air comprimé.

Les besoins de stockage de Gaz de France sont assez considérables. On estime qu'il faut accroître chaque année le volume utile de nos réservoirs souterrains d'une capacité de lordre de $250 \mathrm{mil}$ lions de mètres cubes normaux.

En ce qui concerne les structures aquifères, il y en a dans la région parisienne; celles de Beynes et Saint-llliers sont déjà équipées; on va en équiper une autre à Beynes à quelques $700 \mathrm{~m}$ de profondeur. Il est entendu qu'avant 1976 on doit reconnaitre une structure supplémentaire.

Néanmoins, on arrive un peu à la limite d'utilisation des structures aquifères, ce qui conduit à envisager d'autres techniques de stockage, telles que par exemple, l'utilisation d'engins nucléaires pour créer des cavités de stockage à grande profondeur.

D'autre part, des études sont conduites actuellement en liaison avec ERAP en Bresse pour déterminer les conditions de stockage dans des nappes aquifères sans fermeture, dans des couches horjzontales.

La croissance continue des besoins explique qu'on envisage, dans une décennie environ, l'épuisement des structures les meilleures.

Mais il existe peut-être des structures aquifères dans lesquelles on ne pourra pas mettre de gaz pour des raisons d'environnement. A Beynes, par exemple, il y a une structure qui conviendrait pour stocker de l'air : c'est celle de l'Albien où il y a de l'eau potable; il n'est évidemment pas question d'y stocker du gaz.

On a proposé, rappelle $M$. Chambadal, de faire dans les mêmes sites deux réservoirs superposés, l'un pour le gaz, l'autre pour l'air, bien entendu à des profondeurs différentes.

C'est, en effet, dans certains cas une solution réalisable, répond M. Roux. A Beynes, par exemple, on pourrait stocker dans l'Albien qui doit être à $200 \mathrm{~m}$ de profondeur environ, de l'air; au niveau $400 \mathrm{~m}$, on stockerait du gaz manufacturé; et l'on envisage d'accumuler dans le Séquanien, à $700 \mathrm{~m}$ de profondeur, du gaz naturel : on aurait là trois réservoirs superposés avec trois gaz diflérents.

M. MAscarello estime que la vraie guestion est de savoir s'il ect possible de réaliser - dans dix ou quinze ans - des réservoirs nécessaires à une usine d'accumulation de $2000 \mathrm{MW}$ dans les régions qui intéressent la production et le transport de l'énergie (dans des terrains calcaires, notamment).

Sur une question de M. le Président, M. Roux apporte les informations suivantes

Il y a beaucoup de gisements de sel en France et en Europe: gisement du Valentinais où est implanté le réservoir de Tersanne, immenses gisements salins de Bresse, d'Alsace, de la Région de Manosque, etc.

Mais, la difficulté est de trouver des réservoirs à proximité des centres de consommation d'énergie électrique. C'est peut être la Région lyonnaise qui est la mieux placée par rapport aux gisements salins.

M. Schlumberger (Sté Geostock) donne une idée de l'ordre de grandeur des possibilités dans ce domaine en indiquant que dans les dix prochaines années, on envisage en France de stocker 30 millions de mètres cubes dans des couches de sel.

M. Alllerer appelle l'attention sur ce, qu'au terme de la quinzaine dannées que l'on envisage pour les installations de production de pointe par stockage d'air et turbine à gaz, le problème de localisation près des grands centres de consommation aura perdu de son acuité pour deux raisons:

D'une part, les coûts de transports aux tensions de 760000 ou un million de volts seront devenus sensiblement plus faibles.

D'autre part, il aura probablement été nécessaire de localiser la très grande majorité des centrales de base sur la mer. De ce fait, tout emplacement franchement éloigné de la mer deviendra intéressant au point de vue de la production de pointe, même le Massif Central et le nord des Alpes.

Peut-ĉte cela vaudrait-il la peine de regarder les conjugaisons possibles de stockage d'air avec des réservoirs hydrauliques d'une centaine de mètres de profondeur. Des galeries horizontales creusées à partir du fond de la retenue, moyennant une forme particulière de leur débouché peuvent stocker l'air à pression constante: le « water drive » est disponible et il est peu coûteux de réaliser l'aménagement et la sortie de lair comprimé. On avait même lancé autrefois l'jdée d'une sorte de hangars à l'air, établis au fond d'une cuvette de réservoir; ils auraient seulement à résister à des écarts de pression faibles et à leur poids propre et en sens inversé à la poussée d:Archimède.

M. Charrier observe que les études techniques et économiques ráalisées aujourdhui ont naturellement tendance à prendre comme base les groupes turbines à gaz de faible puissance actuellement réalisés. D'ici quatre ou cinq ans, les constructeurs seront capables de liver des turbines à gaz de 200 à $250 \mathrm{MW}$ fonctionnant en cycle simple et peut-être $500 \mathrm{MW}$ avec des cycles plus sophistiqués. Il serait dommage que la prospective des stations d'accumulation d'ait comprimé fut distordue par la méconnaissance des possibilités à moyen terme des constructeurs.

Sur une question de M. le Président, M. Rigollot précise que les spécialistes estiment possible la réalisation de réservoirs en terrain sédimentaire mais leur prix, toutes choses égales d'ailleurs, sera supéricur à celui du même réservoir établi en bon rocher.

M. RAUn apporte les informations suivantes:

La reconnaissance de craje de Ventetil, près d'Epernay, avait 
conduit E.D.F., il y a quelques années, à ne pas retenir ce site pour la construction d'importants ouvrages souterrains destinés à un aménagement de pompage.

A mon avis, les calcaires ne peuvent convenir à l'accumulation avec compensation hydraulique que s'ils sont de résistance suffisante et si leur régime hydrologique est favorable. Bien que les problèmes soient différents, les informations apportées par la construction des réservoirs pétroliers sont très précieuses dans ce domaine.

Je suis frappé, dit M. Schlumberger de la similitude des problèmes que nous avons à résoudre pour le stockage des hydrocarbures.

En examinant la nature de l'industrie de l'énergie, en France nous arrivons de plus en plus maintenant à trouver, quel que soit l'endroit, la meilleure manière de réussir des stockages souterrains. Nous avons de gros programmes de recherche en ce domaine actuellement et jai bon espoir que nous allons réussir.

M. le Président pense que cette conclusion est applicable aux réservoirs recherchés par E.D.F. et ouvre la discussion sur les problèmes liés à l'exploitation de ces derniers.

Le refroidissement de l'air comprimé avant son injection dans le réservoir exige-t-il des investissements élevés? demande $M$. le Président. Ils sont de l'ordre de 5 ou $6 \%$ de l'investissement par kilowatt installé, répond $M$. Roudier.

Un bref débat - auquel participent M. le Président, M. Plichon, M. Gérard et M. Raud - s'instaure sur le risque d'érosion des parois d'un réservoir souterrain avec compensation hydraulique lorsque l'exploitation de celui-ci se fait, non plus sur une base saisonnière (comme pour les réservoirs de Gaz de France) mais suivant un rythme bi-journalier comme il est envisagé par les producteurs d'énergie électrique. Les observations dans ce domaine semblent inexistantes.

M. RAUD donne l'information suivante:

Dans un réservoir d'aménagement hydropneumatique, Ia vitesse de l'air est maximum lorsque l'eau est à son niveau le plus haut. Compte tenu de ce que l'air est à une pression de 25 bars et a donc une densité d'une trentaine de $\mathrm{kg} / \mathrm{m}^{3}$, j'ai pris une garde limite pour éviter une agitation de la surface de l'eau, la vitesse de l'air à une valeur de l'ordre du mère/seconde. C'est une valeur faible qu'il sera intéressant d'augmenter car elle impose une garde dont l'incidence sur le coût du réservoir n’est pas négligeable.

b) Cycles thermodyamiques et TeChnologie des turbines a gaz:

Sur le choix de la pression à ladmission des turbines à gaz, $\mathbf{M}$. Chambadal fait l'intéressante mise an point ci-après:

Si nous prenons une turbine à gaz ordinaire, et si nous portons en abscisses la pression d'admission c'est-à-dire la pression maximale de l'air (la pression minimale est nécessairement la pression atmosphérique), on sait que le rendement passe par un maximum pour une certaine pression qui est la pression optimale au point de vue du rendement.

Mais il y a un autre optimum de pression au point de vue de la puissance spécifique, c'est-à-dire au point de vue de la puissance par unité de débit dair.

Bien entendu, la solution consiste à adopter un compromis entre ces deux optinums; dans l'exemple donné par $M$. Roudrer on a choisi 9,3 bars; les machines actuelles ont, en effet, des pressions d'admission de l'ordre de 10 bars.

Il serait d'un plus grand intérêt de préciser l'optimum, non pas pour la turbine à gaz, mais pour l'ensemble de l'installation.

Or, dans le coût de cette installation, le réservoir intervient pour une grande part; et nous savons que le réservoir sera d'autant plus économique que son volume sera plus petit, donc que la pression sera plus elevée si bien qu'on aurait intérêt à adopter une pression de lordre de 20 à 25 bars.

Dans ces conditions, si l'on prend une turbine à gaz classique (comme celles de Vitry ou celles d'Alsthom), on doit la compléter par une turbine amont qui détend lair entre 25 et 10 bars afin de ne pas perdre la pression, (donc l'énergie utilisable des gaz) entre 25 et 10 bars. Ainsi, la turbine à gaz devrait normalement comporter une deuxicme chambre de combustion.

C'est là qu'intervient l'injection d'eau... M. DE VILleneuve a signalé, à juste raison, que l'intérêt de l'injection - à première vue, tout au moins - est moins grand dans le cas d'une turbine à gaz combinée avec un réservoir que dans le cas d'une turbine à gaz ordinaire; ceci est vrai, voici pourquoi :

Je suppose que l'injection d'eau augmente la puissance développée par la détente dans la turbine proprement dite, par exemple, de $10 \%$. Dans le cas d'une turbine à gaz classique, comme le compresseur prend, à lui seul, les deux-tiers de la puissance, cela représente un gain de $30 \%$ sur la puissance utile; mais dans le cas où la turbine est couplée avec un réservoir, le gain n'est plus que de $10 \%$ : linjection d'eau est done moins intéressante. Mas ces $10 \%$ se répercutent une fois de plus sur le volume du réservoir, et apportent une économie sur les investissements.

Il est vrai que cette injection d'ean nous fait perdie du rendement, parce que le liquide injecté dans la chambie de combustion décrit un cycle de turbine à vapeur à contre-pression avec un rendement nettement inférieur à celui de la turbine à gaz; donc, le rendement total est diminué.

Il y a un moyen de remédier à cela : c'est de placer à la sortie de la turbine une chaudière de récupération, de manière à injecter dans la chambre de combustion non pas de l'eau liquide, mais de la vapeur qu'on obtient ainsi gratuitement. Dans ces conditions, le gain de puissance est le même que dans le premier cas, mais le rendement augmente au lieu de diminuer. Reste à savoir si lopération est rentable.

Quoi qu'il en soit, il ne semble pas, at theure actuelle, que des cycles très compliqués soicnt justifiés pour ce genre d'installation. L'idée de l'injection d'eau est connue depilis très longtemps, mais les bonnes perspectives actuelles de ce procédé satténueront dans un avenir plus lointain du fait de la tendance à latigmentation des températures d'admission.

A l'heure actuelle, celles-ci atteignent $870^{\circ}$ à Vitry, mais près de $1000^{\circ}$ dans les turbines à gaz Alsthom; dans les prochaines années, il y a de bonnes chances pour que ceue évolution se poursuive.

Mais cette évolution conduit aussi à une évolution de la pression d'admission. Or, à mesure que la température et la pression d'admission augmentent, l'intérêt de linjection diminue. Par conséquent, l'injection d'eau ne présente d'intérêt qu'à l'heure actuelle, c'est-àdire tant que les températures sont encore relativement modérées, mais dans dix ou quinze ans cette technique sera probablement périméc.

Sur la demande de M. le Président. R. Rigotlot donne les informations ci-après sur les cycles mixtes qu'il a étudiés en liaison, avec E.D.F.

Dans l'un des cycles, la turbine à gaz était conjuguée avec une turbine à vapeur entraînant un compresseur dair; aux heures de pointe, le compresseur et le réservoir d'air souterrain alimentent simultanément la turbine à gaz. Nous avons également envisagé d'injecter l'air à une température assez basse dans le réservoir et d'utiliser des refroidisseurs entre les différents étages de compression d'air, afín d'améliorer le rendement de celle-ci.

Nous avons également envisagé de substituer de l'azote à l'aì comme fluide de dilution dans la turbine à gaz. Mais il s'agit là de solutions sophistiquées dont l'utilisation n'est pas prévisible dans un avenir immédiat, mais que l'on pourra examiner à nouveau lorsque les perfectionnements de la technologje permettront à la turbine à gaz de fonctionner à haute température.

M. Mascarialo souligne que le cycle mixte turbine à gaz-turbine à vapeur utilisé à Vitry sur une installation de $320 \mathrm{MW}$ ne constitue qu'un début; d'ailleurs, il a été étudié pour des conditions économiques existant il y a cinq ou six ans (prix élevé du combustible, taux d'intérêt des emprunts relativement modéré) qui se sont modifiées depuis.

M. Mascarello donne ensuite quelques renseignements sur les centrales thermiques $B$ étudiées depuis par E.D.F.

Le cycle avec préchauftage de l'air comburant à la vapeur, utilisé et expérimenté sur les deux cycles mixtes de $320 \mathrm{MW}$ de la centrale de Vitry, adapté aux nouvelles conditions économiques, conduit à une unité de production thermique très simplifiée et originale (thermique $B$ ) nettement moins chère au kilowatt installé que le thermique habituel.

On peut associer à cette unité une turbine à gaz pour créer un cycle mixte. I.intérêt économique de cette association est fonction du niveau de prix des turbines à gaz et des fuels lourds distillés sous vide.

L'adjonction d'un stockage dair comprimé, lorsque cela est possible, à une unité utilisant un cycle mixte est intéressante.

M. Banal observe, à propos des cycles mixtes envisagés dans l'exposé de M. Mascarel. Lo, que le stockage d'air comprimé exige, pour être économique, une pression élevée et ne peut ĉtre réalisé en conservant des turbines à gaz analogues à celles de Vitry.

Ainsi, d'une manière générale, le stockage d'air comprimé exige le développement d'une filière de urbines à gay différente de celle qui se développe pour l'utilisation sans stockage.

M. Charrier souligne la possibilité d'une forte réduction du coût des turbines à gaz dans l'avenir.

Il est important, dit-il, de rappeler qu'en 1.962, les groupes de $5000 \mathrm{MW}$ qui ont fait le succès de la General Electric dans ce domaine étaient construits à l'unité et à la demande du client.

En 1963, la General Electric a pris la décision de lancer uno série, sans savoir si elle allait obtenir des commandes; ipso facto, elle a abaissé ses prix de revient dans d'importantes proportions: alors qu'en 1962 un groupe de $5000 \mathrm{MW}$ était vendu 92 dollars par kilowatt, au bout de deux ans, le prix de la machine est tombé à 
70 dollars par kilowatt; à partir de 1965, pour des lots de quatre groupes, des sociétés «électriques 》 américaines ont pu acheter ce type de machine pour 65 dollars le kilowatt installé.

Ceci montre l'influence de la fabrication de série dans le domaine des turbines à gaz et l'intérêt pour les sociétés « électriques » d'établir des programmes prévisionnels.

Aucun constructeur français ne se lancera dans une politique analogue à celle de la General Electric Co., s'il n'est pas assuré d'avoir un minimum de possibilités de débouchés.

M. le Président fait observer que la turbine à gaz peut se développer dans l'avenir presque indépendamment de l'extension de l'accumulation d'énergie dans des réservoirs d'air comprimé et réciproquement.

11 suggère ensuite de discuter de la question du coût des installations envisagées:

c) Coût des INSTALIATIONS :

M. Rigollor précise que ses études économiques portent sur des turbines différentes de celles actuellement réalisées. Pour les réservoirs établis dans les gisements de sel, il a adopté une pression d'air de 25 bars et une seule "chaufe», contre 40 bars et deux «chaufles » pour les réservoirs en « aquiferes poreux ». Les prix ne peuvent être établis que par analogie avec ceux des groupes déjà construits et dépendent de l'intérêt que les constructeurs portent à ce type de matériel.

A propos de la notion de «flexibilité » des con̂ts des turbines à gaz, dont a parlé M. MASCARELlo, en l'opposant implicitement à la rigidité des coûts du matériel hydraulique, $M$. Ract-Madoux se montre sceptique et remarque que depuls deux ans les estimations du coût des projets d'accumulation pneumatique sont passées de 200 à $600 \mathrm{~F}$ par kilowatt installé.

Il pense cependant qu'une usine d'accumulation pneumatique de
$200 \mathrm{MW}$, implantée en Bretagne, serait compétitive avec une station de même puissance utilisant le pompage hydraulique et constituerait un élément de progrès très important pour les stations à accumulation pneumatique de l'avenir.

Au terme d'un bref débat, il conclut: dans le Massif Central et les Alpes, où on peut disposer de hauteurs de chute assez grandes, le pompage hydranlique l'emportera probablement. Mais, il faut continuer à rechercher - pour la Région parisienne, notamment des réservoirs en terrains sédimentaires, crayeux ou calcaires, susceptibles d'alimenter des usines d'accumulation pneumatique de 1000 ou $2000 \mathrm{MW}$ qui seront nécessaires vers 1980 .

Personne ne demandant plus la parole, M. le Président tire, en ces termes, la conclusion de la séance:

L'accumulation d'énergie par stockage d'air comprimé n'est pas une utopie; c'est une des possibilités dont on dispose aujourd'hui pour produire de l'énergie de pointe.

Il apparaît qu'on peut réaliser les réservoirs souterains nécessaires i cet effet; différentes solutions ont été proposées et M. AILlERET suggère notamment l'utilisation des réservoirs des centrales hydratlliques.

Le matériel «turbine à gaz » existe et peut connaitre des développements très importants si l'on améliore ses performances et son prix.

Par conséquent c'est un domaine dans lequel il est utile de poursuivre les études. Il faut aussi que les utilisateurs de matériel et les constructeurs puissent comparer les performances et les prix de revient de ce mode de production avec d'autres, tels que la turbine à gaz ou le pompage hydraulique.

Maintenant, Messieurs, en votre nom, je remercie vivement les conférenciers et tous ceux qui ont pris part à ce débat et nous ont apporté des informations fort intéressantes.

La séance est levée à 18 heures. 